\title{
Electrochemical Detection of Parts-Per-Billion Lead via an Electrode-Bound DNAzyme Assembly
}

\author{
Yi Xiao, ${ }^{\dagger}$ Aaron A. Rowe, ${ }^{\ddagger}$ and Kevin W. Plaxco*,‡,§ \\ Department of Physics, Materials Department, and Institute for Polymers and Organic Solids, Department of \\ Chemistry and Biochemistry, and Program in BioMolecular Science and Engineering, University of California,
} Santa Barbara, California 93106.

Received October 11, 2006; E-mail: kwp@chem.ucsb.edu

The development of specific ion sensors is linked to pressing needs for the rapid detection of toxic metals. ${ }^{1-4}$ Of particular interest has been the detection of lead $\left(\mathrm{Pb}^{2+}\right)$, an important pollutant with major routes of human exposure arising from lead-based paints and contaminated soils and foodstuffs. ${ }^{5}$ Because of the often severe effects of lead toxicity, which include renal malfunction and the inhibition of brain development, ${ }^{6}$ allowable juvenile serum lead levels are just 100 parts-per-billion (ppb). ${ }^{7}$

Current protocols for the detection of lead require inductively coupled plasma mass spectroscopy (ICP/MS) (see Supporting Information (SI)), a rather complex laboratory technique. Motivated by the desire for rapid, portable means of quantifying low-level lead contamination, recent years have seen the development of fluorescent ${ }^{8}$ and colorimetric ${ }^{9}$ sensors achieving parts-per-billion detection limits. These optical methods, however, suffer from possible drawbacks including potential false signals arising from contaminating colorants, fluorophores and quenchers, and, frequently, a reliance on cumbersome optical equipment. Electrochemical methods, in contrast, benefit from the impressive miniaturization of modern microelectronics, the relative paucity of electroactive contaminants, and the relative stability and environmental insensitivity of electroactive labels and thus are less likely to suffer from these potential drawbacks. ${ }^{10}$ Unfortunately, however, the electrochemical lead detection methods reported to date require complex, multistep protocols involving the reductive deposition of metallic lead followed by anodic stripping voltammetry. ${ }^{10}$ Here we propose a simpler electrochemical approach based on the highly specific, metal-induced activation of a lead-requiring DNAzyme.

DNAzymes are catalytic DNA sequences isolated via in vitro selection. ${ }^{11,12}$ Cofactor-dependent DNAzymes can often be generated from this approach by adding varying cofactors and cofactor concentrations during the selection process. Using a lead-dependent DNAzyme produced by this method, several groups have created optical lead sensors that couple the presence of this cofactor with catalytic activities producing fluorescent or colorometric outputs. 8,9 Here we adapt this same lead-dependent DNAzyme in an electrochemical biosensor that achieves parts-per-billion (nanomolar) sensitivity and excellent selectivity in a single, convenient measurement step.

The $\mathrm{Pb}^{2+}$-requiring DNAzyme we have employed, the " $8-17$ " DNAzyme, is a sequence-specific nuclease acting on a singlestranded DNA substrate containing a single, sessile ribo-adenine (indicated by arrows in Scheme 1). ${ }^{8 \mathrm{a}, 13}$ The sensor consists of a methylene-blue (MB) modified version of this catalytic DNA strand (1) hybridized to its complementary, 20-base substrate oligonucleotide (2). This complex, which is chemi-absorbed to a gold electrode

$\dagger$ Department of Physics, Materials Department, and Institute for Polymers and Organic Solids.

¥Department of Chemistry and Biochemistry.

$\S$ Program in BioMolecular Science and Engineering. via a $5^{\prime}$ terminal thiol on the catalytic strand, ${ }^{14}$ is relatively rigid, presumably preventing the $\mathrm{MB}$ from approaching the electrode to transfer electrons (Scheme 1, left). (Alternatively, transfer may occur through the double stranded DNA from a MB intercalated within the double-stranded regions of the sensing DNA. ${ }^{15}$ ). In the presence of $\mathrm{Pb}^{2+}$, the trans-acting catalytic strand cleaves the sessile phosphodiester of the substrate into two fragments (Scheme 1, middle). These fragments presumably dissociate from the complex, allowing the MB to transfer electrons to the electrode (Scheme 1, right).

Scheme 1. A Schematic of the DNAzyme-Based Electrochemical Sensora

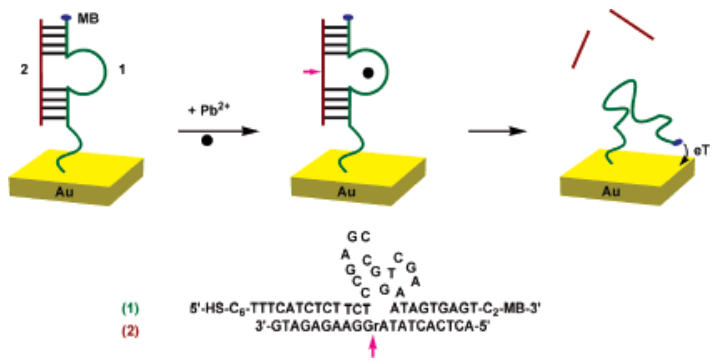

${ }^{a}$ Inset: secondary structure of the " $8-17$ " lead-dependent DNAzyme employed here.

The catalytic strand (1) is composed of a functional domain of 15 deoxynucleotides flanked by $5^{\prime}$ and $3^{\prime}$ substrate-recognition domains of 9 nucleotides each. Previous studies indicate that this represents the optimal compromise between stable complex formation and efficient dissociation after cleavage. ${ }^{11,12}$ Of note, deposition of the catalytic strand during sensor fabrication must be carefully controlled to produce optimal signal gain and reproducibility. As determined by electrochemical measurements, the surface coverage of the MB-modified catalytic strand (1) was maintained within the range of $3.8 \pm 0.3 \mathrm{pmol} \cdot \mathrm{cm}^{-2}$, an electrode loading level at which sensor gain is maximized.

The DNAzyme-based sensor is sensitively and specifically responsive to its target ion (Figure 1,2 ). In the absence of $\mathrm{Pb}^{2+}$ we observe only small, reproducible Faradaic currents. Upon increasing $\mathrm{Pb}^{2+}$ we observe a large increase Faradaic current, saturating at a $\sim 60 \%$ signal increase above $10 \mu \mathrm{M}$. The directly measured detection limit of the current sensor architecture after $1 \mathrm{~h}$ incubation at $37^{\circ} \mathrm{C}$ is $0.3 \mu \mathrm{M}(62 \mathrm{ppb})$, and the signal gain is linear over the range from 0.5 to $10 \mu \mathrm{M}$ (104 to $2070 \mathrm{ppb})$. At approximately $30 \mathrm{~min}$ (see SI, Figure S1) the sensor equilibration time constant is, however, rather slower than that observed for the equivalent solution phase (homogeneous) optical sensor. ${ }^{8,9}$

Control experiments support the proposed sensing mechanism. For example, sensors fabricated using an inactive mutant catalytic strand (3) (due to a $\mathrm{T}$ to $\mathrm{C}$ mutation at position 13 ) ${ }^{10}$ do not respond 

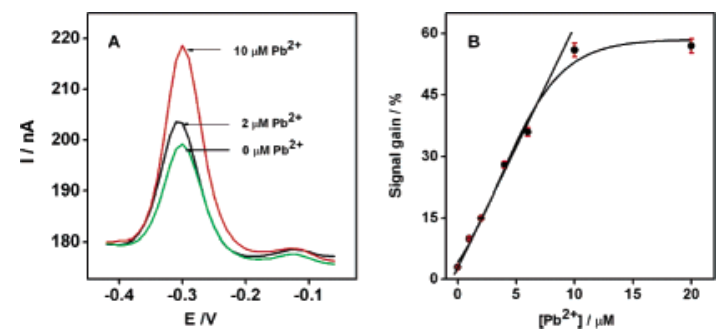

Figure 1. The lead sensor is responsive to its target. (A) Shown are alternating current voltammograms of the sensor obtained after $1 \mathrm{~h}$ incubation at various $\mathrm{Pb}^{2+}$ concentrations. (B) A dose-response curve for the lead sensor. The illustrated error bars represent the standard deviation of four measurements conducted with a single electrode at each concentration; multiple electrodes were used to collect the entire data set. (Relative sensor response (\%), is employed because this is more reproducible electrode-to-electrode than the absolute current change.)
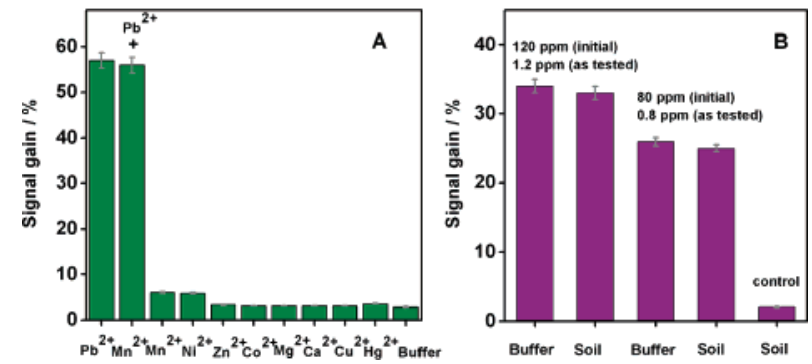

Figure 2. The sensor is both specific (capable of rejecting similar ions) and selective (uninhibited by complex, contaminant-ridden samples). (A) Little signal change is observed when the sensor is challenged with divalent metal ions other than $\mathrm{Pb}^{2+}$ (all at $10 \mu \mathrm{M}$ ). (B) The sensor is selective enough to employ with soil extracts. Shown are the signals obtained after the sensor is challenged with positive control samples comprising lead at 120 or 80 ppm in buffer, with extracts of soil spiked with equivalent amounts of lead, and extracts from a control soil sample lacking detectable lead. All five samples were extracted with acetic acid (to solubilize the lead) using EPA Method 3050B and diluted 100-fold with buffer prior to analysis (i.e., whereas the initial lead concentrations were 120 and $80 \mathrm{ppm}$, the detected levels were 1200 and $800 \mathrm{ppb}$ ).

when challenged with $10 \mu \mathrm{M} \mathrm{Pb}^{2+}$ (see SI, Figure S2). A sensor built using an unreactive, all-deoxyribose substrate strand $(4)^{10}$ also fails to respond when similarly challenged (see SI, Figure S3). It thus appears that the observed increase in electron transfer requires the specific, lead-induced cleavage of the proper DNAzyme.

The specificity of the sensor was determined by challenging it with the divalent metal ions $\mathrm{Mn}^{2+}, \mathrm{Ni}^{2+}, \mathrm{Zn}^{2+}, \mathrm{Co}^{2+}, \mathrm{Mg}^{2+}, \mathrm{Ca}^{2+}$, $\mathrm{Cu}^{2+}$, and $\mathrm{Hg}^{2+}$ at $10 \mu \mathrm{M}$ concentrations (Figure 2A) (see $\mathrm{SI}$, Figures S4-S7). The response to these ions is effectively indistinguishable from the response observed for control samples lacking exogenously added metal ions, and thus the sensor appears to be as specific as the DNAzyme upon which it is based..$^{8,9}$ Likewise the sensor's response to $\mathrm{Pb}^{2+}$ is unaffected by the presence of divalent contaminating ions such as $\mathrm{Mn}^{2+}$ (Figure 2A).

At $62 \mathrm{ppb}(0.3 \mu \mathrm{M})$ the detection limit of our lead sensor is more than sufficient for the routine monitoring of lead levels in food and environmental samples: For example, the U.S. Food and Drug Administration has set an action level of $500 \mathrm{ppb}(2.5 \mu \mathrm{M})$ for lead in products intended for use by infants and children. ${ }^{16}$ Similarly, the U.S. Environmental Protection Agency's (EPA) action levels for total lead in soils range from 100 to 400 parts-per-million (ppm) (0.5 to 1.5 millimolar). ${ }^{17}$ As evidence of the applicability of our sensor for such applications, we have tested its ability to quantify lead in soil samples at parts-per-million concentrations. Using EPA method $3050 \mathrm{~B}^{18}$ to extract the lead from standard soil samples prior to measurement with our sensor we obtain values within a few percent of those determined using standard ICP/MS analysis methods (Figure 2B). The lead in these spiked soil samples (at $80 \mathrm{ppm}$ or $120 \mathrm{ppm}$ ) was extracted in $1 \mathrm{~mL}$ of $10 \%$ HOAc (to solublize the often insoluble lead salts present in soil) before being diluted 100-fold prior to analysis, illustrating the sensor's exceptional sensitivity.

The DNAzyme-based electronic sensor described here exhibits excellent sensitivity and specificity for its target ion. It also offers reasonable selectivity, speed, and operational convenience. Finally, given the potential for in vitro selection to produce cofactordependent DNA-cleaving DNAzymes (for example, both copperand histidine-dependent DNAzymes have been reported with the requisite sequence-specific nuclease activity ${ }^{19}$ ) and given that it appears that DNAzymes can be immobilized without loss of activity, ${ }^{8 c, 20}$ this approach may prove of general utility for electrochemical analyte detection.

Acknowledgment. This work was supported in part by NIH Grant EB002046 and by Lawrence Livermore National Laboratory (URP-06-019). We thank Professors Yi Lu and Dr. Juewen Liu for providing helpful discussion and Professor Alan Heeger for advice and support. We thank Julius Carstens (Oilfield Environmental and Compliance, Inc.) for providing the standard leadcontaining soil samples employed in this research.

Supporting Information Available: The experimental conditions for the preparation of DNAzyme-modified electrodes and for the reported analysis and control experiments. This material is available free of charge via the Internet at http://pubs.acs.org.

\section{References}

(1) Miyawaki, A.; Llopis, J.; Helm, R.; Mccaffery, J. M.; Adams, J. A.; Ikura, M.; Tsien, R. Y. Nature 1997, 388, 882-887.

(2) Walkup, G. K.; Imperiali, B. J. Am. Chem. Soc. 1996, 118, 3053-3054.

(3) Turner, A. P. F. Science 2000, 290, 1315-1317.

(4) (a) Nolan, E. M.; Lippard, S. J. J. Am. Chem. Soc. 2003, 125, 1427014271. (b) Woodroofe, C. C.; Lippard, S. J. J. Am. Chem. Soc. 2003 , $125,11458-11459$

(5) http://www.environment.nsw.gov.au/leadsafe/index.html, 2001.

(6) Needleman, H. L. Human Lead Exposure; CRC Press: Boca Raton, FL, 1991.

(7) http://yosemite.epa.gov/ochp/ochpweb.nsf/content/blood_lead_levels htm, 2003.

(8) (a) Li, J.; Lu, Y. J. Am. Chem. Soc. 2000, 122, 10466-10467. (b) Liu, J. Lu, Y. Anal. Chem. 2003, 75, 6666-6672. (c) Swearingen, C. B Wernette, D. P.; Cropek, D. M.; Lu, Y.; Sweedler, J. V.; Bohn, P. W. Anal. Chem. 2005, 77, 442-448. (d) He, Q.; Miller, E. W.; Wong, A. P. Chang, C. J. J. Am. Chem. Soc. 2006, 128, 9316-9317.

(9) (a) Liu, J.; Lu, Y. J. Am. Chem. Soc. 2003, 125, 6642-6643. (b) Liu, J Lu, Y. J. Am. Chem. Soc. 2004, 126, 12298-12305. (c) Liu, J.; Lu, Y. J. Am. Chem. Soc. 2005, 127, 12677-12683.

(10) (a) Veselova, I. A.; Shekhovtsova, T. N. Anal. Chim. Acta 2000, 413 95-101. (b) Yantasee, W.; Timchalk, C.; Weitz, K. K.; Moore, D. A Lin, Y. Talanta 2004, 67, 617-624. (c) Chow, E.; Hibbert, D. B.; Gooding, J. J. Anal. Chim. Acta 2005, 543, 167-176.

(11) (a) Breaker, R. R.; Joyce, G. F. Chem. Biol. 1994, 1, 223-229. (b) Breaker, R. R. Science 2000, 290, 2095-2096.

(12) (a) Santoro, S. W.; Joyce, G. F. Proc. Natl. Acad. Sci. U.S.A. 1997, 94 , 4262-4266. (c) Brown, A. K.; Li, J.; Pavot, C. M.-B.; Lu, Y. Biochemistry 2003, 42, 7152-7161.

(13) (a) Lu, Y. Chem--Eur. J. 2002, 8, 4588-4596. (b) Faulhammer, D Famulok, M. Angew. Chem., Int. Ed. 1996, 35, $2837-2841$.

(14) Levicky, R.; Herne, T. M.; Tarlov, M. J.; Satija, S. K. J. Am. Chem. Soc. 1998, 120, 9787-9792.

(15) Boon, E. M.; Ceres, D. M.; Drummond, T. G.; Hill, M. G.; Barton, J. K. Nat. Biotechnol. 2000, 18, 1096-1100.

(16) http://www.atsdr.cdc.gov/HEC/CSEM/lead/standards regulations.html, 2006

(17) Identifying Lead Hazards in Your Home, EPA-747-F-96-007; U.S. Environmental Protection Agency: Washington, DC, 1996.

(18) This method is based on similar protocol: Method 3050B, rev. 2; USEPA SW-846, 3rd ed, December 1996.

(19) (a) Carmi, N.; Shultz, L. A.; Breaker, R. R. Chem. Biol. 1996, 3, 10391046. (b) Roth, A.; Breaker, R. R. Proc. Natl. Acad. Sci. U.S.A. 1998 95, 6027-6031.

(20) Wernette, D. P.; Swearingen, C. B.; Cropek, D. M.; Lu, Y.; Sweedler, J. V.; Bohn, P. W. Analyst 2006, 131, 41-47.

JA067278X 